Comment le mal vient aux hommes

\title{
En toute innocence...
}

\section{Michèle Vignaux}

\section{(2) OpenEdition \\ Journals}

Édition électronique

URL : http://journals.openedition.org/shakespeare/1156

DOI : 10.4000/shakespeare. 1156

ISSN : 2271-6424

Éditeur

Société Française Shakespeare

Édition imprimée

Date de publication : 1 novembre 1997

Pagination : 181-193

Référence électronique

Michèle Vignaux, "En toute innocence... », Actes des congrès de la Société française Shakespeare [En ligne], 15 | 1997, mis en ligne le 01 janvier 2007, consulté le 02 mai 2019. URL : http:// journals.openedition.org/shakespeare/1156 ; DOI : 10.4000/shakespeare.1156 


\title{
E N T O U T E I N N O C E N C E ...
}

\author{
Le problème réside précisément en ce \\ qu'il n'est nullement nécessaire d'avoir \\ un cœur mauvais, phénomène assez \\ rare, pour causer de grands maux. \\ La triste vérité est que la plus grande \\ part du mal est faite par des gens qui ne \\ se sont jamais décidés à être bons ou \\ mauvais ${ }^{1}$.
}

I. - or absent - mothers ?

Janet Adelman a récemment consacré un livre à ce qu'elle nomme les , où elle traite entre autres de Gertrude, de Volumnia, d'Hermione, ainsi que de Lady Macbeth, des filles de Lear et de la sorcière bannie Sycorax ${ }^{2}$. Mais il est une catégorie à laquelle on ne s'est guère intéressé, à notre connaissance, la catégorie des mères absentes : celles de Jessica, de Desdémone ou d'Ophélie. Nous nous proposons de suivre ici l'histoire d'Ophélie qui, selon un procédé habituel chez Shakespeare, peut se lire comme une intrigue secondaire qui éclaire l'intrigue principale selon une autre perspective en même temps qu'elle en constitue un commentaire, contribuant à la polyphonie de l'œuvre, et mérite à ce titre la plus grande attention.

D'emblée, Ophélie est montrée comme soumise à un discours exclusivement masculin, celui de son frère puis celui de son père, bien propres à susciter la méfiance et la peur - le mot revient, martelé avec une fréquence 
inquiétante. A l'acte I, scène 3, après quelques brèves paroles d'adieu, Laerte met Ophélie en garde contre les avances d'Hamlet en ces termes :

$$
\begin{aligned}
& \text { Laer. For Hamlet, and the trifling of his favour, } \\
& \text { Hold it a fashion and a toy in blood, } \\
& \text { A violet in the youth of primy nature, } \\
& \text { Forward not permanent, sweet not lasting, } \\
& \text { The perfume and suppliance of a minute, } \\
& \text { No more. }
\end{aligned}
$$

No more but so ?

For nature crescent does not grow alone

In thews and bulk, but as his temples waxes

The inward service of the mind and soul

Grows wide withal. Perhaps he loves you now,

And now no soil nor cautel doth besmirch

The virtue of his will ; but you must fear,

His greatness weighed, his will is not his own,

For he himself is subject to his birth. [...]

Then weigh what loss your honour may sustain

If with too credent ear you list his songs,

Or lose your heart, or your chaste treasure open

To his unmastered importunity.

Fear it, Ophelia, fear it, my dear sister,

And keep within the rear of your affection,

Out of the shot and danger of desire.

The chariest maid is prodigal enough

If she unmask her beauty to the moon.

Virtue itself scapes not calumnious strokes. [...]

Be wary then; best safety lies in fear :

Youth to itself rebels, though none else near.

Oph. I shall th'effect of this good lesson keep

As watchman to my heart. But, good my brother,

Do not, as some ungracious pastors do,

Show me the steep and thorny way to heaven,

Whilst like a puffed and reckless libertine

Himself the primrose path of dalliance treads And recks not his own rede.

\section{O fear me not.}

$(\mathrm{I} .3 .5-51)^{3}$

Survient alors Polonius, qui donne à son fils quelques conseils d'un pragmatisme brutal ; puis, réagissant à une dernière allusion de Laerte à l'adresse d'Ophélie, Polonius demande à cette dernière de lui rapporter les propos de son frère, sur lesquels il renchérit immédiatement : 
Pol. Marry, well bethought.

'Tis told me he hath very oft of late

Given private time to you, and you yourself

Have of your audience been most free and bounteous.

If it be so - as so 'tis put on me,

And that in way of caution - I must tell you

You do not understand yourself so clearly

As it behoves my daughter and your honour.

What is between you ? Give me up the truth.

$O p h$. He hath, my lord, of late made many tenders

Of his affection to me.

Pol. Affection, pooh! You speak like a green girl

Unsifted in such perilous circumstance.

Do you believe his tenders, as you call them ?

$O p h$. I do not know, my lord, what I should think.

Pol. Marry, I'll teach you. Think yourself a baby

That you have ta'en his tenders for true pay

Which are not sterling. Tender yourself more dearly,

Or - not to crack the wind of the poor phrase,

Running it thus - you'll tender me a fool.

(I.3.90-109)

Par l'effet d'une cruelle ironie, lorsqu'Hamlet se trouve à nouveau en présence d'Ophélie, son comportement semble confirmer les pires craintes de Laerte et de Polonius : c'est la fameuse scène dite du cloître (, III.1).

Comme toujours chez Shakespeare, l'intrigue est élaborée avec un soin minutieux. Si l'on suit la chaîne des malentendus entre Ophélie et Hamlet, on peut la faire remonter à ce premier discours de Polonius, à la suite duquel Ophélie perd toute confiance dans ses capacités de jugement, ce qui la conduit à faire le mal, en toute innocence. Lorsqu'Ophélie reparaît sur scène au début de l'acte II, aussitôt après l'entretien de Polonius avec son domestique Reynaldo (à qui il vient de demander d'espionner les faits et gestes de Laerte, ce qui contribue à renforcer l'atmosphère de suspicion généralisée), c'est décomposée par la peur qui, on le sait, est plutôt mauvaise conseillère. Aux questions de son père, elle répond par la vision d'épouvante que l'on sait :

$O p h$. Alas, my lord, I have been so affrighted. Pol. With what, i'th' name of God?

$O p h$. My lord, as I was sewing in my chamber,

Lord Hamlet, with his doublet all unbraced,

No hat upon his head, his stockings fouled,

Ungartered, and down-gyvèd to his ankle,

Pale as his shirt, his knees knocking each other, 
And with a look so piteous in purport

As if he had been loosèd out of hell

To speak of horrors, he comes before me.

(II.1.75-85)

Polonius est prompt à établir un diagnostic, dont il ne démordra plus ; Hamlet est égaré par l'amour:

\author{
Pol. Mad for thy love? \\ My lord, I do not know, \\ But truly I do fear it. [...]
}

Pol. What, have you given him any hard words of late?

Oph. No, my good lord, but as you did command

I did repel his letters, and denied

His access to me.

(II.1.86-110)

Ces instructions de Polonius auxquelles Ophélie fait allusion sont en effet mentionnées par Polonius lui-même à la scène suivante (II.2.140-3), lors de son entrevue avec le roi et la reine, à l'issue de laquelle la décision est prise de Ophélie sur Hamlet, selon l'expression de Polonius (II.2.162) : ce sera la scène du cloître, dans laquelle il est clair qu'Ophélie se laisse manipuler, en toute bonne foi, par obéissance filiale, et avec les meilleures intentions du monde, puisqu'il s'agit de vérifier la sincérité des sentiments d'Hamlet à son égard et que, entre temps, la reine a laissé entendre que leur union serait tout à fait envisageable :
And for your part, Ophelia, I do wish
That your good beauties be the happy cause
Of Hamlet's wildness; so shall I hope your virtues
Will bring him to his wonted way again,
To both your honours.

Polonius lui-même semble avoir renoncé à attribuer des intentions malhonnêtes à Hamlet :

I am sorry that with better speed and judgement

I had not quoted him. I feared he did but trifle

And meant to wreck thee. But beshrew my jealousy !

By heaven, it is as proper to our age

To cast beyond ourselves in our opinions 
As it is common for the younger sort

To lack discretion.

(II.1.112-118)

- quoique son naturel méfiant revienne au galop :

Read on this book,

That show of such an exercise may colour

Your loneliness. We are oft to blame in this,

'Tis too much proved, that with devotion's visage

And pious action we do sugar o'er

The devil himself.

L'obéissance filiale d'Ophélie a déjà été soulignée par deux fois : outre e passage déjà cité (II.1.108), Polonius explique au roi et à la reine que sa fille, sur sa demande, lui a remis une lettre d'Hamlet :

I have a daughter - have whilst she is mine -

Who in her duty and obedience, mark,

Hath given me this.

C'est alors, dans cette entrevue arrangée et sous haute surveillance, qu'Ophélie, s'étant avancée vers Hamlet, après une brève formule de salut qui ne parvient guère à le tirer de son humeur méditative, comme paralysée par la duplicité qui lui est imposée, ne trouve rien de mieux pour poursuivre la conversation que de lui remettre ses lettres ! - sans transition et avec une maladresse qui trahit assez le malaise que suscite en elle l'obéissance aveugle à ce qui est sans nul doute une injonction de son père :

$O p h$. My lord, I have remembrances of yours That I have longèd long to re-deliver. I pray you now receive them.

Ham. No, no, not I. I never gave you aught.

Oph. My honoured lord, I know right well you did, And with them words of so sweet breath composed As made the things more rich. Their perfume lost, Take these again; for to the noble min

Rich gifts wax poor when givers prove unkind. 
Le passage au pluriel de généralité qui vient renforcer le caractère sententieux des derniers vers est bien fait pour déclencher la fureur d'Hamlet, dont assurément, il est impossible de dire qu'il se soit montré envers Ophélie. Jusqu'ici, il avait tout au plus suscité sa frayeur et sa pitié - les deux passions, notons-le au passage, que doit éveiller le héros tragique - , mais nul sentiment d'ingratitude. A tout prendre, ce serait plutôt lui qui aurait lieu d'être offensé par la soudaine froideur d'Ophélie qui, obéissant aux injonctions paternelles a, de façon plus ou moins explicite, repoussé ses avances, vraisemblablement sans raison, et sans doute aussi sans conviction, comme on peut l'inférer du passage déjà cité : II.1.108-110. Ce qui expliquerait qu'Hamlet nie lui avoir fait don de quoi que ce soit, puisque pour lui, il n'y a rien de commun entre la nouvelle et l'ancienne Ophélie ${ }^{4}$. Remarquons en outre qu'Ophélie ne lui témoigne nul ressentiment lorsque, à la scène suivante, lors de la représentation de la pièce-dans-la-pièce, il manifeste son désir de s'asseoir près d'elle, en dépit de la verdeur de ses propos, et surtout de la violence et de la cruauté avec lesquelles il l'a traitée à la scène précédente - peut-être du fait que sa violence était dirigée autant contre lui-même que contre elle, et manifestait un réel désespoir (notons que ce passage est en prose) ; peut-être aussi sa position en porte-à-faux lui causet-elle confusément quelque remords.

Kerrigan fait remarquer que dans cette scène, Hamlet a à l'égard d'Ophélie la même fonction que celle de Gertrude vis-à-vis de lui-même : lui faire perdre les illusions propres à la jeunesse ${ }^{5}$ - à ceci près toutefois que Gertrude l'avait fait de façon involontaire, peut-on dire, du simple fait de son remariage hâtif, tandis que lui le fait sciemment, animé d'une fureur destructrice. On pourrait d'ailleurs appliquer à son attitude envers Ophélie les mots qu'il prononce à l'adresse de Gertrude (III.4.167), lorsqu'il lui dit qu'il ne l'a jamais aimée, et qu'elle a été bien naïve de croire à ses déclarations (III.1.1179 et 121-132) - propos démentis dans la scène du cimetière, qui ne laisse aucun doute quant à l'authenticité de ses sentiments à l'égard d'Ophélie : (V.1.264). Il n'en reste pas moins que le comportement d'Hamlet, malgré toutes ses bonnes intentions, aura des conséquences - directes et indirectes fatales pour Ophélie. La fin du passage révèle à quel point il a été contaminé par le discours ambiant, comme l'indique le pluriel de généralité : thou wilt needs marry, marry a fool ; for / wise men know well enough what monsters you make of them (III.1.139-141). En même temps, son discours extrêmement critique envers lui-même témoigne d'une désillusion profonde sur l'humanité, dont il ne s'excepte d'ailleurs pas :

Get thee to a nunnery. Why, wouldst thou be a breeder of sinners? I am myself 
indifferent honest, but yet I could accuse me of such things that it were better my mother had not borne me. I am very proud, had not borne me. I am very proud,
revengeful, ambitious, with more offences at my beck than I have thoughts to put them in imagination to give them shape, or time to act them in. What should such fellows as I do crawling between heaven and earth? We are arrant knaves all. Believe none of us. Go thy ways to a nunnery.

(III.1.122-130)

Le sommet de la cruauté est sans doute atteint quelques lignes plus loin avec la de Hamlet à Ophélie, en forme de malédiction :

If thou dost marry, I'll give thee this plague for thy dowry: be thou as chaste as ice, as pure as snow, thou shalt not escape calumny.

(III.1.136-138)

La critique se divise en deux tendances sur les motivations de Hamlet dans son rejet d'Ophélie : une première tendance, illustrée entre autres dans l'édition de Dover Wilson pour Cambridge en 1934, considère que Hamlet a dû surprendre les propos de Polonius et de Claudius et leur intention de lui tendre un piège, dont Ophélie serait l'instrument consentant; ce qui expliquerait l'horreur qu'elle lui inspire ${ }^{6}$. Cette hypothèse, qui peut paraître séduisante, reste cependant purement théorique, faute de pouvoir être étayée par la tradition dramaturgique ${ }^{7}$. La seconde tendance, illustrée par exemple par Harold Jenkins (édition Arden), considère que l'horreur inspirée à Hamlet par la conduite de sa mère est une raison suffisante pour expliquer, par contamination, son propre comportement à l'égard d'Ophélie. Quoi qu'il en soit, il reste qu'Ophélie est utilisée par son père, plus ou moins à son insu, soit sous couvert de vérifier les sentiments d'Hamlet à son égard, soit sans qu'on lui ait donné d'explication, mais de toute façon sans que sa volonté ait une part active à l'acte pour le moins moralement douteux qu'on lui fait commettre - ce qui est certainement suffisant pour alerter l'intuition, toujours en éveil, d'Hamlet.

On s'est par ailleurs demandé quels étaient au juste ces péchés don s'accuse Hamlet. La réponse n'est pas facile à trouver, ou même à inférer, du texte ; c'est donc sans doute qu'elle importe assez peu, et de même en ce qui concerne le père d'Hamlet (I.5). Il s'agit plutôt des péchés en général, ceux que 
commet inévitablement tout être humain, et qui sont mentionnés précisément pour signifier l'humanité des personnages; de ceux qui font dire à Hamlet, lorsque Polonius lui affirme qu'il va traiter les comédiens selon leur mérite: (II.2.519-20). Il y a chez lui une sorte d'indulgence générale pour le genre humain qui va de pair avec la violence de son ressentiment à l'égard de ses proches, c'est-à-dire ceux qui le font souffrir. Notons que seuls sont dotés de cette capacité à pécher, outre Hamlet lui-même, son père, sa mère, et peut-être Ophélie; mais ni Horatio (qui n'est malgré tout qu'un personnage secondaire), ni Rosenkrantz et Guildenstern (trop insignifiants), ni Polonius, qui ressemble plutôt à une marionnette se prenant pour le marionnettiste. Reste Claudius, qui est bien sûr capable de péché, mais non - et c'est ce qui le différencie de Gertrude - de repentir.

La scène qui suit le rejet d'Ophélie par Hamlet - et ce n'est sans doute pas le fait du hasard - produit une sorte d'apaisement, que Hamlet trouve auprès des comédiens, et surtout dans l'amitié d'Horatio. Or, que lui dit Hamlet ?

II.

Ce qu'Hamlet admire chez Horatio, et lui envie, c'est sa capacité à s'abstraire de la souffrance que procurent les émotions mal maîtrisées :

$$
\text { thou hast been }
$$

As one, in suff'ring all, that suffers nothing,

A man that Fortune's buffets and rewards

Hath ta'en with equal thanks; and blest are those

Whose blood and judgement are so well commingled

That they are not a pipe for Fortune's finger

To sound what stop she please. Give me that man

That is not passion's slave, and I will wear him

In my heart's core, ay, in my heart of heart

As I do thee.

(III.2.60-69)

L'alliance heureuse de à laquelle Hamlet fait allusion évoque bien sûr la division tripartite de l'âme en intellect, passions et appétits héritée de Platon et courante à la Renaissance. Les questions de la raison, de son exercice dans le jugement, et des relations difficiles qu'elle entretient avec les passions et les appétits, méritent qu'on s'y attarde quelque peu.

Cet équilibre réussi entre la passion et la raison met à l'abri des coups de la Fortune, ou du moins évite d'en souffrir. Malgré les abondantes réflexions qui ont valu à Hamlet sa célébrité romantique, il est aussi un homme d'action, passionné et impulsif dès qu'il cesse de penser, et il est profondément mû non 
pas par la raison, mais par la triple passion de la vérité, de la justice et de la vengeance $^{8}$. A cinq ou six reprises, il se laisse aller à des actes impulsifs, emporté par sa passion : lorsqu'il suit le fantôme de son père, bravant toutes les exhortations à la prudence de ses compagnons (I.5.81); dans la scène du cloître, lorsqu'il donne libre cours à sa fureur envers Ophélie ; lorsqu'il tue Polonius; lorsqu'il lit la missive dont sont porteurs Rosenkrantz et Guildenstern et qu'il les envoie à la mort ; lorsqu'il saute dans la tombe d'Ophélie et défie Laerte ; lorsque, défiant ses propres pressentiments, il décide de se rendre au match d'escrime qui lui sera fatal. Hamlet oscille entre impulsivité et méditation sans parvenir, sinon avec les plus grandes difficultés et seulement à la fin de la pièce, après avoir fait souffrir et tué beaucoup de monde, à trouver le point d'équilibre qui lui permettrait de produire une action qui soit le résultat d'une délibération.

D'autre part, la conception tripartite de l'âme informe les relations d'Hamlet tant avec Ophélie qu'avec Gertrude, quoique de façons différentes : ce qu'Hamlet reproche à Gertrude, explicitement, est de se laisser guider par ses appétits les plus bas ; ce qu'il reproche à Ophélie, implicitement, est son incapacité à penser, c'est-à-dire à s'émanciper par son jugement de la tutelle désastreuse - de son père, ce qui ne lui laissera d'autre issue, après la perte de son amour et la mort de son père, que la fuite dans la folie et finalement dans la mort. Aux yeux d'Hamlet, l'incapacité de Gertrude à établir une distinction entre ses deux maris est le signe irréfutable d'un auto-aveuglement, d'une prostitution de l'esprit, qui à son tour contamine la volonté, c'est-à-dire l'instance de décision: (III.4.80). L'analyse du Mistère d'Adam, jeu de Noël de la fin du XIIe siècle, qui constitue l'un des chapitres du livre d'Erich Auerbach, Mimesis, peut apporter un éclairage sur les relations d'Hamlet avec Gertrude et avec Ophélie : on y voit que que le mal arrive non pas tant par la femme que par la relation de l'homme à la femme, ou en d'autres termes par ce qui, en l'être humain, est de l'ordre du non-rationnel. Dans un premier temps en effet, Adam s'en tient scrupuleusement au rôle que Dieu lui a confié : il doit gouverner Ève par la raison. S'interrogeant sur ce qui le pousse ensuite à céder à la tentation, Auerbach commente

il balance entre l'angoisse et le désir - non pas exactement le désir de la pomme mais le désir de s'affirmer: un homme doit-il craindre là où une femme n'hésite pas [Ève vient de croquer la pomme] ? Et quand il surmonte enfin son angoisse et prend la pomme, il accomplit le geste avec une pensée extrêmement touchante : ce que sa femme a fait, il veut le faire aussi, il veut avoir confiance en elle. [... seule Ève] pouvait arriver à ce résultat (avec l'aide du diable), car elle seule est unie à Adam d'une 
manière si particulière que ses actions éveillent en

lui un écho qui le bouleverse ${ }^{9}$.

On ne peut nier que, en dépit de toutes ses fautes, Gertrude est accessible aux raisons que lui fait voir Hamlet. Dans la seconde partie de la pièce, après l'exil d'Hamlet, elle devient une sorte de substitut de la mère dont Ophélie est dépourvue. Enfin, elle se rallie sans ambiguïté à Hamlet à partir du retour inattendu de ce dernier et jusqu'à la fin de la pièce ${ }^{10}$.

Le sort d'Ophélie quant à lui illustre parfaitement l'étouffement de la pensée dont parle Freud: ${ }^{11}$. Dans Eichmann à Jérusalem (1966), H. Arendt a longuement analysé ce mystère stupéfiant que constitue ce qu'elle nomme la d'Eichmann et son incapacité à sortir du rôle qu'il s'était donné, celui de grand criminel de guerre, dans lequel il fonctionnait parfaitement. Elle y revient dans ses Considérations morales :

Il savait que ce qu'il avait alors considéré comme un devoir était à présent appelé un crime, et il acceptait ce nouveau code pénal comme un nouveau langage, sans plus. A sa provision d'expressions toutes faites, passablement limitée, il en avait ajouté quelques nouvelles et était complètement perdu lorsqu'il devait affronter une situation à laquelle aucune d'elles ne s'appliquait [...].

Les clichés, les phrases toutes faites, l'adhésion à des codes d'expression ou de conduite conventionnels et standardisés, ont socialement la fonction reconnue de nous protéger de la réalité, de cette exigence de pensée que les événements et les faits éveillent en vertu de leur existence. Si nous répondions tout le temps à cette exigence, nous serions très rapidement épuisés; avec Eichmann, c'était différent : de toute évidence, il ne connaissait pas une telle exigence. (p. 26-7)

Penser est indifféremment dangereux pour toutes les croyances et, par soi, n'en crée aucune nouvelle. Toutefois, la non-pensée, qui semble une attitude tellement recommandable en politique et en morale recèle aussi quelques dangers. En prévenant de l'examen et de ses dangers, elle enseigne aux gens à $s$ 'attacher fermement a tout ce que peuvent etre les règles de conduite prescrites par telle époque, dans telle société. Ce à quoi ils s'habituent est moins le contenu de règles, dont un examen serré les plongerait dans l'embarras, que la possession de règles sous lesquelles puissent être subsumés des cas 
particuliers. En d'autres termes, ils sont habitués à

ne jamais se décider. (p. 54-5)

La pensée, présente à l'état virtuel chez tout être humain mais exercée par bien peu, est l'attribut d'Hamlet, et le place dans une sorte d'absolu ; l'amour a la même fonction en ce qui concerne Ophélie, puisqu'elle ne peut survivre à sa perte, que l'on considère son sentiment pour Hamlet, comme ses chansons nous y invitent sans ambiguité, ou son amour filial. Ils sont, chacun dans son ordre, des êtres d'exception, peu adaptés à la vie dans une société moyenne, à moins que ce soit la société qui ne puisse tolérer de tels individus ${ }^{12}$. Mais avec leur mort - et c'est là en définitive la marque de toute tragédie $^{13}$ - le monde autour d'eux a perdu quelque chose d'irremplaçable, comme dans Roméo et Juliette.

Imaginons un instant un Hamlet en comédie ou en romance ${ }^{14}$ supposition qui, après tout, n'est pas forcément si saugrenue, tant il est vrai que l'œuvre de Shakespeare a acquis un statut de patrimoine commun, qui en fait un matériau sur lequel l'esthétique post-moderne se plaît à bâtir. Que l'on songe seulement aux adaptations d'Edward Bond (Lear [1971]), d'Aimé Césaire

(Une Tempête [1974]), et pour nous rapprocher de Hamlet, à Tom Stoppard (Rosencrantz and Guildenstern are Dead [1967], mais aussi son mini-Hamlet [1976], version condensée de quinze minutes pour sept acteurs, faite d'un collage de répliques de la pièce de Shakespeare) ou à Salman Rushdie (le conte intitulé dans le recueil East, West [1994]). Si l'on envisage les potentialités comiques de Hamlet, la lutte entre les générations pourrait bien être plus pertinente que la lutte entre les sexes. Polonius en vieux barbon de comédie, Claudius en usurpateur-fornicateur, Gertrude en mère souillée, induisent chez Ophélie et Hamlet des comportements qui ne sont pas de leur âge, alors que dans une comédie, les valeurs qu'ils incarnent auraient pu triompher, comme c'est le cas dans Le Songe d'une nuit d'été. Il doit être possible d'imaginer une Gertrude non seulement revenue de son aveuglement et repentante, mais favorisant d'une manière ou d'une autre l'assassinat de Claudius (à moins qu'il ne meure de mort naturelle), à qui succèderait un Hamlet libre de s'unir à Ophélie sous l'œil finalement satisfait de Polonius...

Michèle VIGNAUX Université de Versailles-St Quentin 


\section{${ }^{N}$ O T E S}

${ }^{1}$ Hannah Arendt, Considérations morales, traduction française Marc Ducassou et Didier Maes, Paris, Rivages, 1996, respectivement p. 34 et 58 .

${ }^{2}$ Suffocating Mothers. Fantasies of Maternal Origin in Shakespeare's Plays, Hamlet to The Tempest, Routledge, New York et Londres, 1992, dont le chapitre traitant de Hamlet est repris dans l'édition extrêmement utile de la pièce, assortie d'un panorama critique, due à Susanne L. Wofford (Bedford Books of St. Martin's Press, Boston et New York, 1994, p. 256-82).

${ }^{3}$ Les références renvoient à l'édition établie par G. R. Hibbard (O.U.P., 1987).

${ }^{4}$ William Kerrigan voit dans la scène (relatée par Ophélie en II.1.75-85) où Hamlet scrute en silence le visage d'Ophélie à la recherche d'un indice quelconque qui lui permettrait de comprendre les raisons de cette soudaine transformation, une sorte de prologue en forme de pantomime à la grande scène dite de la chambre à coucher (closet scene) entre Hamlet et Gertrude à l'acte III (Hamlet's Perfection, Johns Hopkins University Press, Baltimore et Londres, 1994, p. 64). Pour la thèse selon laquelle la violence d'Hamlet est à la mesure du désarroi et de l'isolement dans lesquels le plonge la défection - qu'il perçoit comme une trahison - d'Ophélie, voir John Dover Wilson, What Happens in Hamlet (1935), trad. fr. Dominique Goy-Blanquet, Pour comprendre Hamlet, Paris, Seuil, 1992, p. 122-153.

${ }^{5}$ Op.cit., p. 100-101.

${ }^{6}$ Le passage clé pour déterminer cette question se situe à l'acte II, scène 2, selon que l'on fait entrer Hamlet au vers 159 c'est l'option retenue par Dover Wilson, dont il donne les raisons dans Pour comprendre Hamlet, p. 122-153 - ou au vers 167 option plus courante, adoptée par Harold Jenkins (Arden, 1982), Philip Edwards (Cambridge, 1985), G.R. Hibbard (Oxford, 1987).

Voir à ce sujet la note de Philip Edwards (éd. Cambridge) à l'acte II, sc. 2, v. 159 ; ainsi que la note de G.R. Hibbard (éd. Oxford) à II.2.167. 
${ }^{8}$ La remarque judicieuse d'Hannah Arendt, que (op. cit., p. 34), invite à nuancer le jugement romantique à l'encontre d'Hamlet. Par ailleurs, H. Arendt rappelle utilement la distinction médiévale entre d'une part la méditation, qui ne vise pas à la délibération, bien que parfois, mais rarement, elle y aboutisse, et d'autre part la délibération qui, elle, est supposée avoir une finalité concrète (p. 47).

${ }^{9}$ Mimesis : la représentation de la réalité dans la littérature occidentale [1946], traduction française Cornélius Heim, Paris, Gallimard, 1968,p. 153-160 ; la citation se trouve p. 160.

${ }^{10}$ Sur cette question, le textedu premier in-quarto de 1603 , si imparfait par ailleurs, apporte une lumière précieuse, comme l'a montré de façon convaincante Philip Edwards dans son édition Cambridge de 1985, p. 8-32. Voir également Thomas Clayton, ed The "Hamlet" First Published Q1, 1603 : Origins, Forms, Intertextualities, Newark, U. of Delaware Pr., 1992 ainsi que, pour un exposé succint et très clair, l'introduction de Susanne L. Wofford à son édition de Hamlet (v. réf. ci-dessus, n. 2), p. 16-7.

${ }^{11}$ Freud, Nouvelles Conférences sur la psychanalyse (1932), éd. fr. Gallimard (1936), 1978, p. 226. Freud poursuit quelques lignes plus loin en appelant de ses vœux la : «Puisse un jour l'intellect - l'esprit scientifique, la raison - accéder à la dictature dans la vie psychique des humains! Tel est notre vœu le plus ardent. La raison - sa nature même nous en est garante - ne négligera pas de donner aux sentiments humains et à tout ce qu'ils déterminent la place qui leur est due».

${ }^{12}$ Cf. Richard Marienstras, Le Proche et le Lointain, Paris, éditions de Minuit, 1981, à propos d'Othello et de Desdémone, p. 196-199.

${ }^{13}$ Cf. Northrop Frye, Fools of Time, Toronto, 1967, p. 5-6 : «The dialectic of tragedy works through to a situation in which the heroic is normally dead and the less heroic is all that can remain alive. Tragedy often ends with the survivors forming, or about to form, a secondary or social contract, a relation among more ordinary men which will achieve enough working justice or equity to minimize further tragedy. It usually expresses some limiting or falling away of perspective after the great heroic voices have been silenced», et Richard Marienstras, op. cit., p. 210-211. 
${ }^{14}$ On sait que ce genre se caractérise entre autres par des retrouvailles générales, toutes générations confondues. 